\title{
Kidney Stone Protocols to Reduce Emergency Department Overcrowding
}

\author{
Shital Shah, Dino P Rumoro, Mollie Pillman, Samuel Hohmann \\ Shital Shah, PhD, Department of Health Systems Management, Rush University \\ Dino P. Rumoro, DO, MPH, FACEP, Department of Health Systems Management and \\ Department of Emergency Medicine, Rush University
}

Mollie Pillman, MS-HSM, Department of Health Systems Management, Rush University \& Society of Diagnostic Medical Sonography

Samuel Hohmann, PhD, UHC \& Department of Health Systems Management, Rush University

Corresponding author:

Shital_C_Shah@rush.edu

Shital Shah, PhD, Department of Health Systems Management, Rush University

\begin{abstract}
Background: As emergency department (ED) overcrowding affects timely patient care, strategies such as standardize protocols are required.

Objective: The purpose of this study was to 1) investigate impact of utilizing various Computed Tomography (CT) scans modalities, namely CT without contrast (WO), with contrast (W), and with and without contrast (WWO) in diagnosing kidney stones on ED operational metrics (i.e., CT order to report time, room to discharge, and ED length of stay), and 2) develop, implement, and test kidney stone protocols.

Methods: The study included both retrospective, cross-sectional, and pre/post analyses of patients presenting to the ED of a large, academic medical center with suspicion of kidney stone that received CT scans from December 2010 to December 2011. ANOVA, t-test, Chi-Square test, and linear regression were used for statistical analyses.

Results: During pre-implementation, statistically significant difference of at least 41.7 minutes was observed between CT WO and CT WWO for all operational metrics. After implementation of the kidney stone protocol, (i.e., switching to CT WO contrast) the order compliance rate improved from $35.9 \%$ to $72.8 \%$, with similar savings for CT order to report times.

Conclusions: With $37 \%$ additional patients evaluated using CT WO and $3 / 4$ to 1 hour savings in CT order to report time, the estimated net capacity gain is 32 to 43 routine ED patients for a 50,000 volume ED. Thus, standardization of protocol using the developed framework for other common diseases (e.g., abdominal pain) may add ED capacity and revenue while reducing ED overcrowding issues.
\end{abstract}

Keywords: Overcrowding; Kidney Stone; Protocols; CT Scans; Emergency Department 


\section{Introduction}

\subsection{Background}

Due to the economic conditions and increasing overall demand for services, Emergency Departments (EDs) are experiencing higher volumes while simultaneously having to scale back available resources, leading to overcrowding, which is detrimental to both the facility and the patient (Burt \& McCaig, 2006). To combat this growing problem, EDs are examining operational processes that influence overall length of stay (LOS), as well as front-end, throughput, and backend (Nash, Zachariah, Nitschmann, \& Psencik, 2007)-8; (Terris, Leman, O'Connor, \& Wood, 2004); (Wilson \& Nguyen, 2004); (Oakley \& Braitberg, 2005); (Marsh, Anderson, Bastani, \& Bastani, 2004/10); (King, Ben-Tovim, \& Bassham, 2006);(Kinsman et al., 2008) strategies for eliminating unnecessary practices that add time and cost to the patient's visit. EDs are analyzing LOS (i.e., throughput) in relation to common diseases in order to ensure that they are following best operational and clinical practices and eliminating unnecessary treatments whenever possible. One example of a condition that was found to have differentiations in practice and potential for time and cost savings is urolithiasis (kidney stone disease) (Graham, Luber, \& Wolfson, 2011).

Kidney stone affects approximately 5\% of United States population, with the majority of patients typically seeking initial care in the EDs (Westphalen, Hsia, Maselli, Wang, \& Gonzales, 2011). In 2009, there were over 1.3 million ED visits (i.e., 441 per 100,000 people) for kidney stones across United States (Foster, Stocks, \& Borofsky, 2012). Although in many cases the stones pass naturally, oftentimes they do not, which can cause patients extreme pain, leading them to seek treatment in the ED (Graham et al., 2011). When a patient is suspected of having kidney stones based on his or her symptoms (e.g., dehydration, swelling of the kidneys, severe pain in back, side, stomach or groin, fever, and nausea) (MedlinePlus), a physician will order one of many possible types of radiological tests, namely ultrasound (US), Intravenous Urography (IVU), Helical Computed Tomography (CT) with or without contrast, or Magnetic Resonance Imaging (MRI), to confirm the condition. In recent years, there is a significant trend towards CT scans for accessing patients with suspicion of kidney stones (Westphalen et al., 2011). A major differentiator in CT scans diagnosing involves the administration of contrast dye prior to the CT, with options being defined as CT With Contrast (W), Without Contrast (WO), and Both With and Without Contrast (WWO). The dye, which requires additional evaluation of kidney function through the use of a blood test prior to administration, adds cost and risk to the procedure as well as delays between the room and discharge points in the patient's ED visit. Based on Agency for HealthCare Research and Quality (AHRQ) guidelines developed in association with American College of Radiology (ACR), CT scans WO contrast is most preferred diagnostic test (high specificity [98\%], sensitivity [95\%-96\%]) for flank pain with suspicion of stone disease followed by Intravenous Urography (Agency for Healthcare Research and Quality, 1995 (revised 2011)). In the guidelines, utilization of CT scan W contrast is recommended in case of uncertain diagnosis; the decision to utilize it is left to clinical judgment of physicians. With no recommendations on strategies for guideline implementation as well as liberal use of diagnostic testing (Pines, 2009) (i.e., either to increase accuracy, reduce misdiagnosis 
rates and avoid malpractice litigation, expedite post test clinical decision-making or increase ED throughput pressures) uniform protocols for diagnosing kidney stones in EDs are lacking. This in turn affects ED throughput and operational metrics, resulting in ED overcrowding.

\subsection{Importance}

Decreasing ED beds, increasing ED volumes, and limiting capability to transfer ED patients contribute to ED overcrowding that most EDs across the country are experiencing. A 2009 study estimated the national annual cost of diagnosing and treating kidney stone disease to be approximately $\$ 2.1$ billion (Lotan, 2009). Even with 1-hour reduction in ED stay for these patients, potential for cost savings is substantial. It also underscores a need for the standardization and implementation of protocols for kidney stones, as well as other common diagnoses; using best practices will minimize variation and contribute to reducing length of stay, increasing safety, and alleviating overcrowding.

\subsection{Goals of This Investigation}

The purpose of this study was to 1) investigate impact (i.e., comparative effectiveness) of utilizing various CT scan modalities namely CT WO contrast, W contrast, and WWO contrast in diagnosing kidney stones on emergency department (ED) operational metrics (e.g., throughput times and length of stay), 2) develop and implement kidney stone protocols, and 3) test the effects of protocol implementation on operational outcomes. Evaluating impact of other kidney stone diagnostic modalities such as ultrasound and Intravenous Urography were beyond the scope of this study.

\section{Methods}

\subsection{Study Design and Setting}

The study was conducted in a large, private, non-profit academic medical center (AMC) (Rush University Medical Center, Chicago IL) in a metropolitan area with 672 staffed inpatient beds that includes roughly 200 general medicine beds. The AMC had 34 ED beds with annual ED census of over 50,000 patients. The majority of patients seen in the ED represent acuity of Levels 2, 3 , and 4. The ED sees approximately 500 patients per year with suspicion of kidney stone. The initial study is a retrospective, cross-sectional analysis of all ED patients with suspicion of kidney stone who received CT scans (W, WO, and WWO) in Radiology from December 27, 2010 through August 31,2011 . The second analysis consists of a pre/post study after implementation of a new ED protocol, with the data range prior to September 2011 as the pre timeframe and September 1, 2011 to December 11, 2011 as post timeframe. The study was reviewed by the Institutional Review Board prior to data abstraction and expedited approval was obtained.

\subsection{Selection of Participants}

The sample population (pre- and postintervention) consisted of all patients (i.e., including adult and pediatric) who presented to the ED from December 27, 2010 through December 11, 2011 and were subsequently sent to Radiology for an abdominal or pelvic CT scan based on a suspicion of kidney stones. Data was obtained through abstraction from ED operational reports from the institution's data warehouse as well as from Radiology CT order detail reports, which were effectively merged with patient medical record data to select patients based on their exam type, location, symptoms, and disposition. Total available sample size was 20,847 from the initial reports, but this was 
reduced to 4,065 by selecting only ED patients with abdominal or pelvic CT tests. Finally, the reason for the exam as documented by board certified ED physicians was clinically vetted using keywords (e.g., kidney stone, renal stone, calculi, hemaruria, flank pain, nephrolithiasis, and renal colic among others) to filter only patients for whom stone suspicion or kidney stone symptoms was documented. For data integrity purposes, 15 cases were excluded from the population because their disposition statuses were either

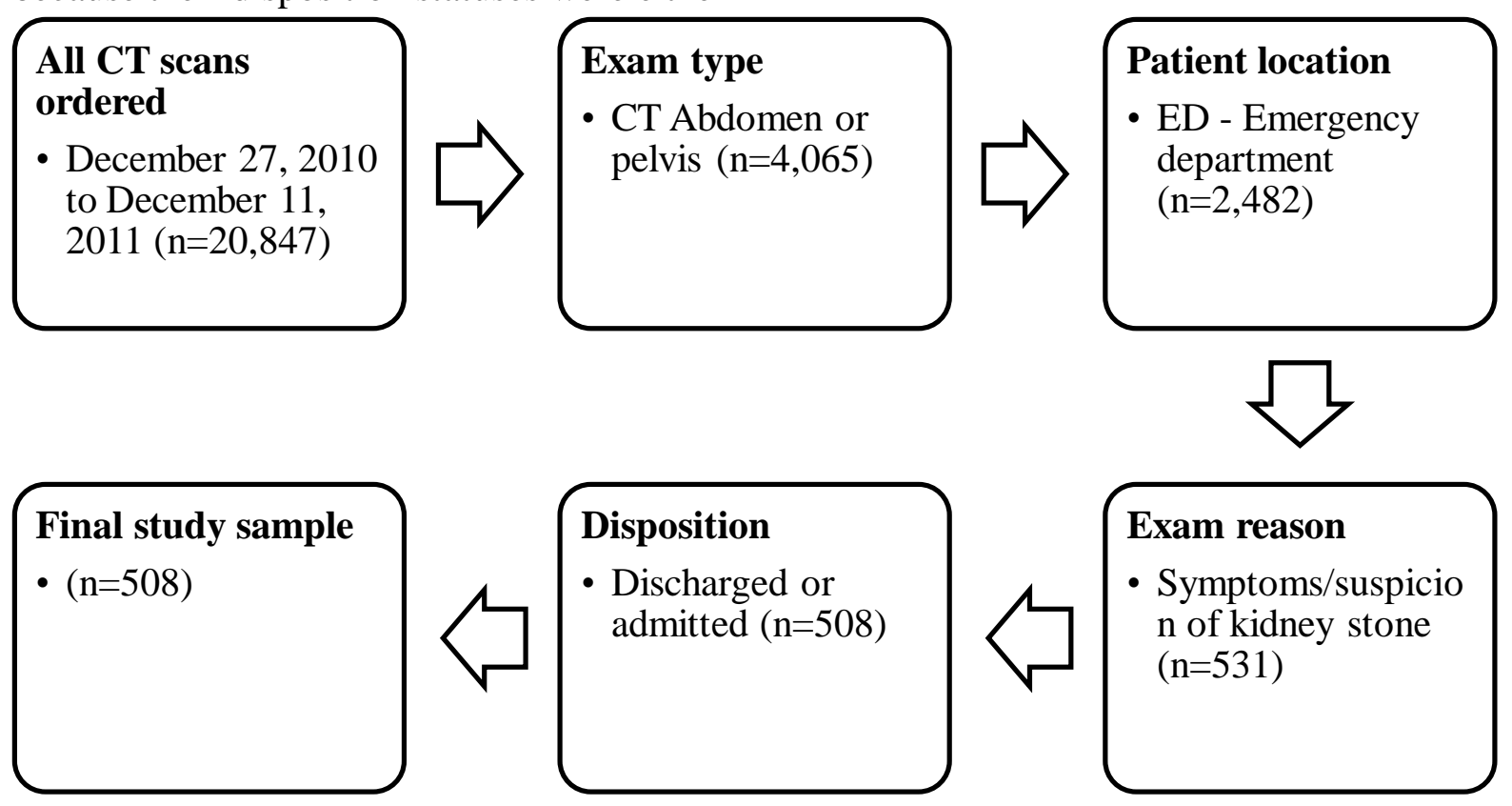

"Observation" (i.e., they led to a longer LOS that was not necessarily associated with the type of CT being ordered) or "Against Medical Advice" (i.e., they led to a shorter LOS than anticipated if patient had stayed). A sample size of at least 172 was needed in order to detect moderate differences in operational outcomes for CT scan patients, assuming a power of $90 \%$ and type I error rate of 5\%. This requirement was met with a final study sample of 508 suspected kidney stone patients (Figure 1).

\section{All CT scans}

December 27, 2010

to December 11 , $2011(n=20,847)$

\section{Final study sample \\ - $(\mathrm{n}=508)$}

Figure 1: Isolation of Population

\subsection{Interventions}

A preliminary analysis was conducted to measure the operational effect of noncontrast CT scans in comparison with those W or WWO contrast, using data from December 27, 2010 through August 31, 2011. In August 2011, the Emergency Department protocols were changed to encourage physicians to order CT scan WO contrast for patients with suspicion of kidney stone(s). The kidney stone protocols were communicated to physicians through email, departmental meetings, and peer-topeer communication. The order compliance rate and operational outcomes were tracked and analyzed for the post implementation timeframe.

\subsection{Methods and Measurements}

Consolidation of the two data sources was conducted by use of a unique ID (accession number) that tied back to the patient's medical record number for identification. Patients with multiple visits were identified and were manually filtered to include those 
ED visits associated with kidney stone symptom criteria and having a CT order within the specified date range. Finally, the database was de-identified and the fields for analysis were formatted and standardized.

Key variables considered include exam contrast type (independent; categorical variable), CT order to report time, room to discharge time, and length of stay (LOS) (continuous dependent variables). Patient demographics (gender [binary], age [continuous]) and medical information (acuity [categorical], ED disposition [binary]), as well as general Emergency Department operating data (day of week [categorical], time of shift [categorical]) were also analyzed as moderating factors. The potential impacts of intervention on patient outcomes were analyzed using information such as whether the patient revisited within 30 days to the $\mathrm{ED}$, as well as revisit diagnosis and subsequent ED disposition.

\subsection{Analysis}

Data was analyzed using Microsoft Excel 2007 (Redmond, WA) and IBM SPSS Statistics (Armonk, New York). Descriptive statistics were generated and the data were checked for normality. To perform the analysis, 2-sided independent t-tests, ANOVA with post hoc Bonferroni, and correlation tests were conducted to test the hypothesis that there was no difference in CT order to report time, room to discharge time, or ED LOS based on CT scan modalities (i.e., W, WO, and WWO). A Type I error rate of 5\% was used for all tests of statistical significance. Following the bivariate tests, linear regressions were performed to test the impact CT scan modalities on outcome measures after controlling the effects of moderating variables. During the pre/post study phase, chi-square test was utilized to evaluate the effects of the implementation of a kidney stone protocol intervention. In addition, all operational metrics were statistically reevaluated for the post implementation timeframe. A multi-site comparison for frequency of various CT scan modalities based on charge descriptions as well as rough estimates of ED LOS for confirmed kidney stone cases was conducted using UHC's clinical database (CDB/RM) ${ }^{\mathrm{i}}$.

\section{Results}

\subsection{Characteristics of Study Subjects}

Between December 27, 2010 and December 11, 2011, there were 508 ED patients with suspicion of kidney stones that received abdominal or pelvic CT scans in the radiology department. Of these, $108(21.3 \%)$ were admitted to the hospital and 400 $(78.7 \%)$ were discharged. Males comprised $42.7 \%$ (217 patients) of the sample population. The average age of the patients was 45.3 years. ED operational data showed Tuesdays and Wednesdays to have the highest volumes of kidney stone patients, and both the first (7am-3pm) and second (3pm-11pm) shifts were busy and served significantly more patients than the third shift. As $94.3 \%$ of patients were of acuity level 3 upon arrival, acuity was disregarded for further analysis (Table 1).

In conducting the pre/post analysis, there were 357 patients $(70.3 \%)$ that were presented prior to the implementation of kidney stone protocol in August 2011, while $151 \quad(29.7 \%)$ visits were postimplementation (Table 1). Between the two

\footnotetext{
' UHC is a cooperative of 116 non-for-profit academic medical centers and 272 of their affiliated hospitals, and its purposes include pooling their patient visit information to support performance improvement. The CDB/RM is a discharge database that encompasses all ED visits and characteristics of the patients' visits, including all billed charges during the visit.
} 
timeframes, there was no statistical difference in terms of gender, age, day of the week, or time of shift, indicating homogeneity between pre- and postimplementation patients. The ED disposition was not significant $(\mathrm{p}=.0950)$, with $83.4 \%$ of patients in the post-implementation group being discharged, as opposed to $76.8 \%$ in the pre-implementation group. It was also shown that prior to September 2011, 203 patients $(56.9 \%)$ received orders for CT scans WWO contrast, while 128 patients $(35.9 \%)$ received WO contrast and 26
(7.3\%) received W contrast. This significantly changed during postimplementation timeframe, where 110 patients $(72.8 \%)$ received WO contrast, only 23 patients $(15.2 \%)$ received WWO contrast, and 18 patients $(12 \%)$ received $\mathrm{W}$ contrast. There was no difference in operational metrics (CT order to report time, room to discharge, or ED LOS) between the pre- and post-implementation groups.

Table 1. Pre- and Post-Implementation Descriptive Analysis

\begin{tabular}{|c|c|c|c|}
\hline Variable Name & $\begin{array}{l}\text { Pre-Population } \\
(\mathrm{n}=357)\end{array}$ & $\begin{array}{l}\text { Post-Population } \\
(\mathrm{n}=151)\end{array}$ & $\begin{array}{l}\text { Difference } \\
\text { Significant? }\end{array}$ \\
\hline $\begin{array}{l}\text { Gender } \\
\text { Male } \\
\text { Female }\end{array}$ & $\begin{array}{l}147(41.2 \%) \\
210(58.8 \%)\end{array}$ & $\begin{array}{l}70(46.4 \%) \\
81(53.6 \%)\end{array}$ & No $(p=0.281)$ \\
\hline $\begin{array}{l}\text { Exam Contrast } \\
\text { Without Contrast (WO) } \\
\text { With Contrast (W) } \\
\text { Both With and Without (WWO) }\end{array}$ & $\begin{array}{l}128(35.9 \%) \\
26(7.3 \%) \\
203(56.9 \%)\end{array}$ & $\begin{array}{l}110(72.8 \%) \\
18(11.9 \%) \\
23(15.2 \%)\end{array}$ & $\begin{array}{l}\text { Yes }(p<.001) \\
\text { Significant shift } \\
\text { from WWO to } \\
\text { WO }\end{array}$ \\
\hline $\begin{array}{l}\text { Day of Week } \\
\text { Sunday } \\
\text { Monday } \\
\text { Tuesday } \\
\text { Wednesday } \\
\text { Thursday } \\
\text { Friday } \\
\text { Saturday }\end{array}$ & $\begin{array}{l}48(13.4 \%) \\
55(15.4 \%) \\
54(15.1 \%) \\
62(17.4 \%) \\
48(13.4 \%) \\
51(14.3 \%) \\
39(10.9 \%)\end{array}$ & $\begin{array}{l}19(12.6 \%) \\
19(12.6 \%) \\
25(16.6 \%) \\
25(16.6 \%) \\
20(13.2 \%) \\
22(14.6 \%) \\
21(13.9 \%)\end{array}$ & No $(p=0.950)$ \\
\hline $\begin{array}{l}\text { Acuity } \\
1 \text { (Highest) } \\
2 \\
3 \\
4 \\
5 \text { (Lowest) } \\
\end{array}$ & $\begin{array}{l}0(0.0 \%) \\
7(2.0 \%) \\
341(95.5 \%) \\
9(2.5 \%) \\
0(0.0 \%) \\
\end{array}$ & $\begin{array}{l}0(0.0 \%) \\
6(4.0 \%) \\
138(91.4 \%) \\
7(4.6 \%) \\
0(0.0 \%) \\
\end{array}$ & No $(p=0.185)$ \\
\hline $\begin{array}{l}\text { Time of Shift } \\
07: 00-14: 59 \\
\text { 15:00-23:59 } \\
00: 00-06: 59\end{array}$ & $\begin{array}{l}151(42.3 \%) \\
132(37.0 \%) \\
74(20.7 \%)\end{array}$ & $\begin{array}{l}72(47.7 \%) \\
56(37.1 \%) \\
23(15.2 \%)\end{array}$ & No $(p=0.304)$ \\
\hline $\begin{array}{l}\text { ED Disposition } \\
\text { Discharged } \\
\text { Admitted }\end{array}$ & $\begin{array}{l}274(76.8 \%) \\
83(23.2 \%)\end{array}$ & $\begin{array}{l}126(83.4 \%) \\
25(16.6 \%)\end{array}$ & No $(p=.092)$ \\
\hline $\begin{array}{l}\text { Age (years) } \\
\text { ED Length of Stav (minutes) }\end{array}$ & $\frac{45.6 \pm 16.7}{395.5+1718}$ & $\frac{44.7 \pm 16.7}{385.4+154.1}$ & $\begin{array}{l}\text { No }(p=0.580) \\
\text { No }(p=0.534)\end{array}$ \\
\hline Room to Discharge Time (minutes) & $343.0 \pm 155.4$ & $332.3 \pm 140.2$ & No $(p=0.463)$ \\
\hline CT Order to Report Time (minutes) & $166.3 \pm 70.2$ & $159.1 \pm 67.2$ & No $(p=0.280)$ \\
\hline
\end{tabular}




\subsection{Main Results}

During the pre-implementation timeframe, there was no statistical difference between CT scan type (i.e., W contrast, WO contrast, or WWO contrast) and gender, age, day of the week, and time of shift. Conversely, disposition status was a significant factor ( $\mathrm{p}<.05)$, with those receiving CT scan W contrast (13 patients, 50\%) were more likely to be admitted than WO contrast (31 patients, $24.2 \%$ ), or WWO contrast (39 patients, 19.2\%) (Table 2).

For the pre-implementation timeframe, ANOVA with post hoc Bonferroni tests revealed $\mathrm{CT}$ order to report time for $\mathrm{CT}$ scan. WO contrast was statistically significant compared to $\mathrm{W}$ and WWO contrast and took on average 97.8 and 44.3 minutes less than CT scan $\mathrm{W}$ and WWO contrast, respectively (Figure 2). The CT order to report time for WWO contrast was 53.5 minutes on average shorter than W contrast (Table 2). There was no statistically significant difference in room to discharge or ED LOS for different CT modalities. However room to discharge time for CT scan WO contrast was 25.6 and 34.6 minutes less than CT scan $\mathrm{W}$ and WWO contrast, respectively indicating practical difference that could still contribute to performance improvement.

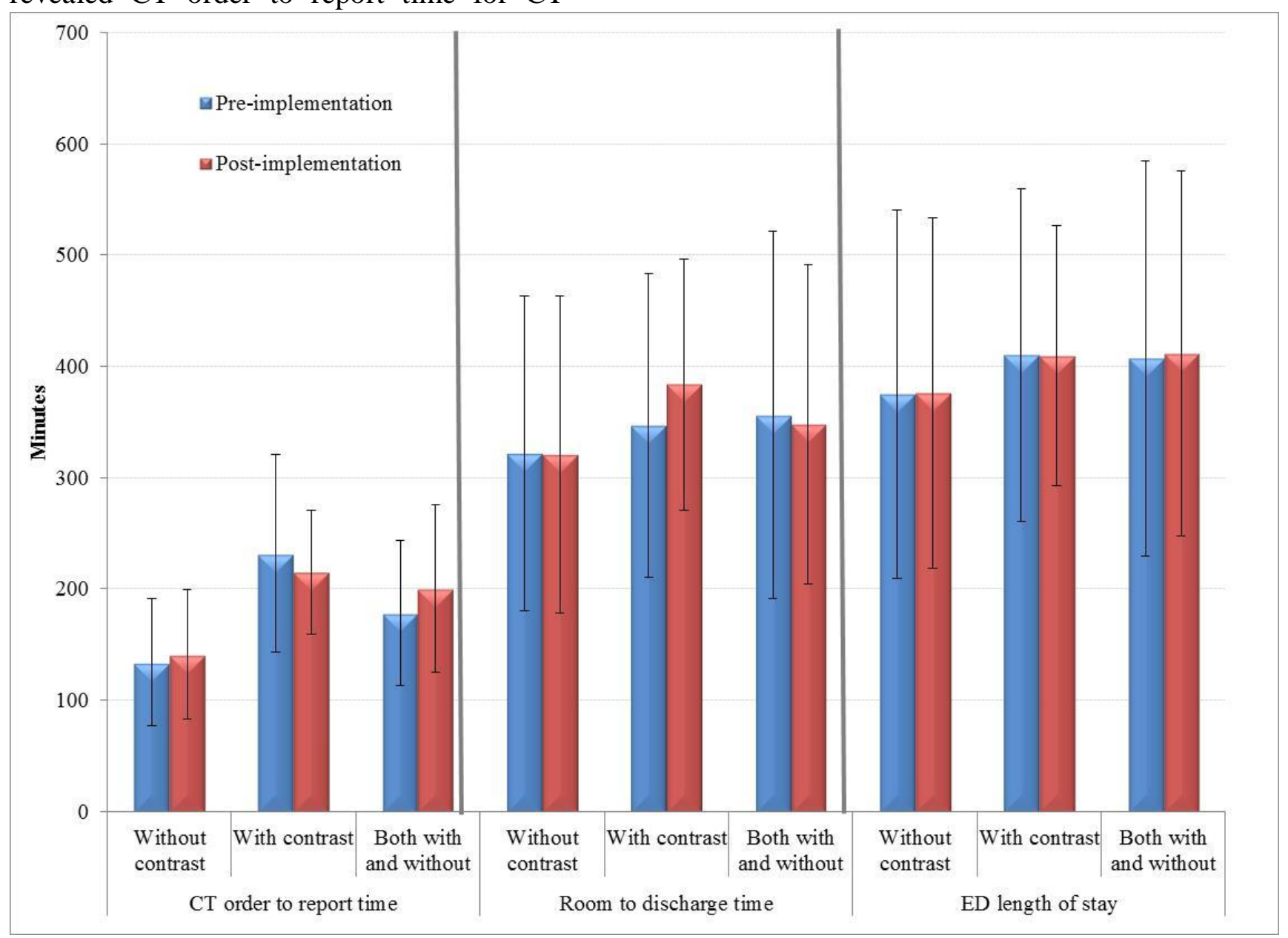

Figure 2: Emergency Department Operational Metrics by CT Scan Order Type 
Table 2. Pre-Implementation Descriptive Analysis by CT Scan

Exam Type

\begin{tabular}{|c|c|c|c|c|c|c|c|}
\hline \multirow[b]{2}{*}{ Variables } & \multicolumn{7}{|c|}{ CT Scan Exam Type } \\
\hline & \multicolumn{2}{|c|}{$\begin{array}{l}\text { Without Contrast } \\
(\mathbf{n}=128)\end{array}$} & \multirow{2}{*}{\multicolumn{2}{|c|}{$\begin{array}{l}\text { With } \\
\text { Contrast } \\
(n=26)\end{array}$}} & $\begin{array}{l}\text { Without } \\
\text { Contrast } \\
(\mathbf{n}=110)\end{array}$ & \begin{tabular}{|l|} 
With \\
Contrast \\
$(\mathbf{n}=18)$
\end{tabular} & $\begin{array}{l}\text { Both With } \\
\text { and Without } \\
(n=23)\end{array}$ \\
\hline & \multicolumn{2}{|c|}{ Pre Implementation } & & & \multicolumn{3}{|c|}{ Post Implementation } \\
\hline Female, \#(\%) & $78(60.9 \%)$ & & $53.8 \%)$ & $118(58.1 \%)$ & $78(60.9 \%)$ & $14(53.8 \%)$ & $118(58.1 \%)$ \\
\hline Age, Avg (Stdev) & $44.7(17.6)$ & 52 & $(17)$ & $45.3(16)$ & $43.3(16.5)$ & $48.2(16.8)$ & $48.3(17.1)$ \\
\hline $\begin{array}{l}\text { ED Disposition - Admitted, } \\
\#(\%) *\end{array}$ & $31(24.2 \%)$ & & $50 \%)$ & $39(19.2 \%)$ & $17(15.5 \%)$ & $3(16.7 \%)$ & $5(21.7 \%)$ \\
\hline $\begin{array}{l}\text { Time of Day } \\
\text { Shift I, \#(\%) } \\
\text { Shift II, \#(\%) } \\
\text { Shift III, \#(\%) } \\
\end{array}$ & $\begin{array}{l}49(38.3 \%) \\
56(43.8 \%) \\
23(18 \%) \\
\end{array}$ & & $\begin{array}{l}42.3 \%) \\
42.3 \%) \\
5.4 \%)\end{array}$ & $\begin{array}{l}91(44.8 \%) \\
65(32 \%) \\
47(23.2 \%) \\
\end{array}$ & $\begin{array}{l}48(43.6 \%) \\
43(39.1 \%) \\
19(17.3 \%) \\
\end{array}$ & $\begin{array}{l}13(72.2 \%) \\
3(16.7 \%) \\
2(11.1 \%)\end{array}$ & $\begin{array}{l}11(47.8 \%) \\
10(43.5 \%) \\
2(8.7 \%)\end{array}$ \\
\hline $\begin{array}{l}\text { Day of Week } \\
\text { Monday, \#(\%) } \\
\text { Tuesday, \#(\%) } \\
\text { Wednesday, \#(\%) } \\
\text { Thursday, \#(\%) } \\
\text { Friday, \# }(\%) \\
\text { Saturday, \#(\%) } \\
\text { Sunday, \#(\%) }\end{array}$ & $\begin{array}{l}16(12.5 \%) \\
18(14.1 \%) \\
24(18.8 \%) \\
18(14.1 \%) \\
20(15.6 \%) \\
15(11.7 \%) \\
17(13.3 \%)\end{array}$ & & $\begin{array}{l}5.4 \%) \\
3.1 \%) \\
9.2 \%) \\
1.5 \%) \\
.7 \%) \\
.8 \%) \\
9.2 \%)\end{array}$ & $\begin{array}{l}35(17.2 \%) \\
30(14.8 \%) \\
33(16.3 \%) \\
27(13.3 \%) \\
29(14.3 \%) \\
23(11.3 \%) \\
26(12.8 \%)\end{array}$ & $\begin{array}{l}15(13.6 \%) \\
16(14.5 \%) \\
19(17.3 \%) \\
13(11.8 \%) \\
16(14.5 \%) \\
18(16.4 \%) \\
13(11.8 \%)\end{array}$ & $\begin{array}{l}3(16.7 \%) \\
4(22.2 \%) \\
3(16.7 \%) \\
3(16.7 \%) \\
1(5.6 \%) \\
2(11.1 \%) \\
2(11.1 \%)\end{array}$ & $\begin{array}{l}1(4.3 \%) \\
5(21.7 \%) \\
3(13 \%) \\
4(17.4 \%) \\
5(21.7 \%) \\
1(4.3 \%) \\
4(17.4 \%)\end{array}$ \\
\hline $\begin{array}{l}\text { CT Order to Report Time, Avg } \\
\text { (Stdev)* }\end{array}$ & $134(57.2)$ & 23 & $8(89.0)$ & $178.3(65.6)$ & $141.2(58.2)$ & $215.3(55.6)$ & $200.4(74.9)$ \\
\hline $\begin{array}{l}\text { Roomed to Discharge Time, Avg } \\
\text { (Stdev) }\end{array}$ & $321.5(141.4)$ & & $.1(136.7)$ & $356.1(165)$ & $320.5(142.4)$ & $383.8(112.8)$ & $347.9(143.8)$ \\
\hline ED LOS, Avg (Stdev) & $374.8(165.7)$ & & $1(149.7)$ & $406.6(177.5)$ & $376(157.5)$ & $409.6(116.7)$ & $411.6(164.1)$ \\
\hline
\end{tabular}

* Statistically significant at 5\% level of confidence. 
Regression analysis for CT order to report time showed that after controlling for other moderating factors, 45.7 minutes time savings could be achieved for each patient that were to shift from receiving WWO to WO contrast for their CT exam (Table 3). The day of week and time of shift analysis indicate patients spend less time in the ED on Saturdays (by 34.4 minutes) and Sundays (by 41.1 minutes) as compared to Monday and during third shift (by 33.8 minutes) as compared to second shift. Based on regression analysis, admitted patient took 103.5 and 89.9 minutes longer than discharged patients for the room to discharge time and ED LOS metrics, respectively. In addition, room to discharge time and ED LOS became significantly shorter by at least 41.7 for CT scan WO contrast compared to WWO contrast after controlling for ED disposition, day of the week and time of the day.

Table 3. Regression Analysis

\begin{tabular}{|c|c|c|c|c|c|c|}
\hline \multirow[b]{2}{*}{ Variables } & \multicolumn{3}{|c|}{ Pre Implementation } & \multicolumn{3}{|c|}{ Post Implementation } \\
\hline & $\begin{array}{l}\text { CT } \\
\text { Order to } \\
\text { Report } \\
\text { Time }\end{array}$ & $\begin{array}{l}\text { Roomed to } \\
\text { Discharge } \\
\text { Time }\end{array}$ & ED LOS & $\begin{array}{l}\text { CT } \\
\text { Order to } \\
\text { Report } \\
\text { Time }\end{array}$ & $\begin{array}{l}\text { Roomed to } \\
\text { Discharge } \\
\text { Time }\end{array}$ & ED LOS \\
\hline Adjusted $R^{2}$ & $21.3 \%$ & $8.1 \%$ & $9.5 \%$ & $27.1 \%$ & $16.3 \%$ & $12.7 \%$ \\
\hline Without Contrast & $-45.7 *$ & $-41.8 *$ & $-41.7 *$ & $-54.8 *$ & -15.5 & -20.4 \\
\hline With Contrast & $42.7 *$ & -44.3 & -35 & 9.9 & 30.8 & 0.4 \\
\hline $\begin{array}{l}\text { ED Disposition - } \\
\text { Admitted }\end{array}$ & 14.2 & $103.5 *$ & 89.9* & -8.5 & $150.1 *$ & 126.1* \\
\hline Tuesday & -6 & 5.6 & -1.9 & 15 & 32.2 & 34.7 \\
\hline Wednesday & -10.7 & -5.6 & -35.6 & 23.4 & 27.7 & 26.7 \\
\hline Thursday & -20.6 & -11 & -51.8 & 0 & -1.4 & -15.7 \\
\hline Friday & -17.5 & -13.8 & -41.4 & 1.6 & -21.2 & -56.8 \\
\hline Saturday & $-34.4 *$ & 17.4 & -42.1 & -25.2 & -37.8 & -88.1 \\
\hline Sunday & $-41.1 *$ & -34.7 & $-92.2 *$ & $-46.1 *$ & -14.9 & -49.7 \\
\hline Shift I & -5.7 & -16.2 & $-48.2 *$ & 11.7 & 32 & -18.8 \\
\hline Shift III & $-33.8^{*}$ & $-47.9 *$ & $-99.9 *$ & -19.1 & 42.8 & -36 \\
\hline Male & 7.5 & $33 *$ & 17.9 & -4.9 & -12.1 & -24.8 \\
\hline Age & 0.4 & 0 & 0.2 & 0.5 & 0.5 & 0.5 \\
\hline Slope & $182.2 *$ & $346.4 *$ & $453 *$ & $179.9 *$ & $278.4 *$ & 401.5* \\
\hline
\end{tabular}

Note: table cells provide beta coefficients along with statistical significance status.

* Statistically significant at 5\% level of confidence. Control groups were with and without contrast, ED disposition Discharge, Monday, Shift II, and female.

During the post analysis (data from September 1, 2011 and later), there was no difference in exam contrast type based on gender, age, day of the week, time of shift, or disposition. During post implementation, the percent of patients with suspicion of kidney stone receiving CT scan WO contrast doubled (i.e., 72.8\%) as compared to pre implementation timeframe (Table 1). CT order to report was significantly shorter by 74.1 and 59.2 minutes for those receiving CT scan WO contrast as compared to $\mathrm{W}$ and WWO contrast, respectively. Based on ANOVA and Post hoc bonferroni results, room to discharge and ED LOS were not statistically significant but indicated practical savings of at least 27.4 minutes for 
CT scan WO contrast compared to $\mathrm{W}$ and WWO contrast. Based on regression analysis, CT order to report time was statistically significant with 54.8 minutes shorter for WO contrast as compared to WWO contrast after controlling for other variables (Table 3). Room to discharge and ED LOS indicated a much longer wait of 150.1 and 126.1 minutes respectively for admitted patients. According to UHC data on confirmed kidney stone patients who were discharged from the ED from a peer group of 51 academic medical centers, approximately $88 \%$ of patients received CT

WO contrast. These patients had an average LOS of 5.8 hours, and the remainder receiving $\mathrm{W}$ or WWO had an average LOS of 6.5 hours. Hospital admissions from the ED were not included in the study sample. In the current study timeframe a total of 46 discharged patients returned to the ED within 30 days of whom only 12 patients ( 5 pre-implementation and 7 postimplementation) returned due to kidney stone related symptoms (Figure 3). During the post implementation, majority of the revisit patients with kidney stone symptoms (i.e., six of seven) were discharged.

\section{Total within 30 \\ day ED re-visit \\ $60(11.8 \%)$ \\ $60(11.8 \%)$}

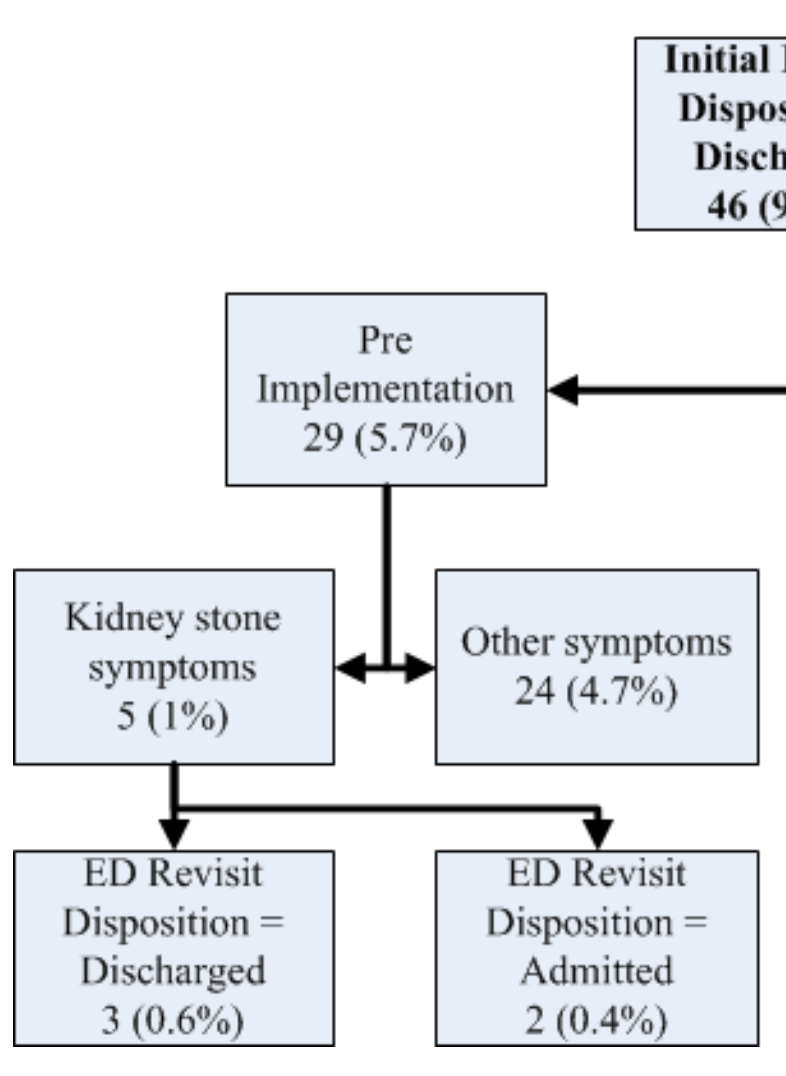

Figure 3: 30 day Emergency Department Revisit for Kidney stone patients.

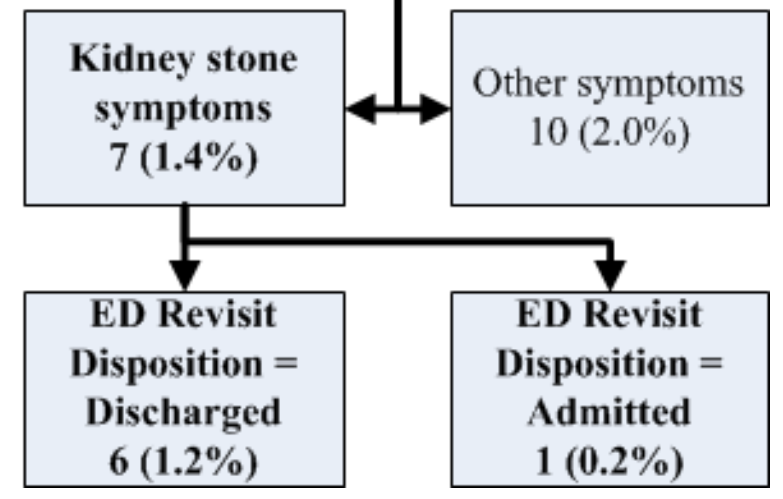

\section{Limitations}

A limitation to this study was that clinical outcomes were out of scope, so the relationship between time savings and clinical effectiveness was not examined. 
Another limitation was the variability among reasons for ordering the CT studies, as this is a free-text field in the radiology order, which had to be filtered manually for purposes of the study. It was possible that a few cases could have been missed using this approach, and in the future, the use of CPT or DRG codes to identify the patient population may lead to more systematic inclusion or exclusion. However, the investigators are confident that at least $95 \%$ of cases were correctly identified and included in the study population.

\section{Discussion}

The underlying result of the analysis showed major improvement in operational outcomes based on using WO contrast instead of $\mathrm{W}$ or WWO, particularly for CT order to report time (i.e., potential time savings of approximately 45 minutes to one hour). This was expected due to the elimination of a blood test prior to CT scans, which would add to the time necessary to perform the CT scan.

Although during the pre-implementation timeframe, time savings for WO contrast as compared to WWO contrast were not significant for more general time measures (i.e., room to discharge and ED LOS) on bivaraite level, they were significant after controlling for ED disposition in the regression analysis. Admitted patients in general took longer for room to discharge and ED LOS than discharge patients indicating different underlying patterns for the two groups. Admitted patients who received WO contrast as compared to $\mathrm{W}$ or WWO contrast had lower room to discharge time and ED LOS most likely due to clearer and definitive diagnosis of kidney stones. Discharge patients had no statistically significant difference in room to discharge time and ED LOS indicating CT order to report time savings were not translated into overall time savings and bottleneck shifted from test ordering to somewhere downstream in the system (i.e., between CT report and ED disposition). Possible causes of these bottlenecks could be clinical uncertainty regarding patients' condition, physician workload, lack of communication when test results were available, bed availability, or even pain management techniques if a patient needed additional care between CT report time and disposition. To address downstream bottlenecks between CT report time and ED disposition, additional interventions such as 1) alerting (i.e., paging, utilization of voice communication systems, EMR links) charge nurse with CT scan results, who will triage them and contact ED physician for quick treatment and ED disposition decision making and 2) changing pain medication protocols toward long-lasting medications can be further investigated. Even in the absence of additional interventions, practical savings of about 27 minutes were seen for room to discharge time and ED LOS, which would still have an impact on overall ED operations and allow for more patients to be seen and treated.

Another significant finding was that patients receiving WWO contrast were shown to have a high likelihood $(80.5 \%)$ of discharge, indicating potentially lower severity than admitted patients as well as the possibility that too much time and testing is used on this population. The fact that WWO contrast was actually shorter than $\mathrm{W}$ contrast in many cases could indicate provider practice of not performing with contrast portion of the order if confirming diagnosis was made after without contrast test. With this practice in place, physicians probably felt comfortable ordering WWO contrast as it was all-inclusive liberal testing approach. As this essentially converts the test order from WWO to just WO contrast with additional lab test, there is a need for standardization 
and tighter application of protocols to avoid unnecessary lab testing and reduce cost and improve operational outcomes.

With the implementation of kidney stone protocols in August 2011, patients were significantly more likely to receive orders for CT WO contrast (i.e., 72.8\%), exhibiting a major shift of approximately $37 \%$ of patients who previously would have most likely received CT WWO contrast. The results indicate higher ED physician compliance towards AHRQ and ACR guidelines of utilizing $\mathrm{CT}$ scans WO contrast (Agency for Healthcare Research and Quality, 1995 (revised 2011)). The change in clinical practice was mainly attributed to a combined strategy of topdown as well as peer-to-peer communication.

Based on UHC data on confirmed kidney stone patients who were discharged from the ED, approximately $88 \%$ of patients received CT WO contrast, which was significantly higher than our observed post implementation numbers. As the data utilized in the current study includes patients with both confirmed and suspected kidney stone as well as admitted patients, the sample is more inclusive; which explains the difference between UHC and the study data.

Though the focus of the study was not on clinical outcomes, the available guidelines and literature, as well as the data in the study, indicates that the use of CT scans WO contrast may not result in adverse clinical impact on the patients. The AHRQ and ACR guidelines for detecting kidney stones is CT scans WO contrast as it provides high specificity (98\%) and sensitivity (95-96\%) (Agency for Healthcare Research and Quality, 1995 (revised 2011)). In addition, the protocols implemented at the AMC were only recommendations to use CT scans WO contrast. The physicians still had the option to order CT scans WWO or CT scans W contrast during the post timeframe. As per Table 1, physicians ordered CT scans WWO or CT scans W contrast during post implementation for $27.15 \%$ (i.e., 41 cases) of time based on their clinical judgement. In addition, during the pre-intervention timeframe, the CT scans WWO had shorter CT order to report time, suggesting the likelihood that only the WO contrast portion of the test was performed. The UHC multisite data also indicates that $88 \%$ of the kidney stone patients had CT scan WO contrast test ordered, indicating that it is also a preferred kidney stone diagnostic test across organizations. Finally, in the post timeframe, only seven patients returned within 30 days to the ED with recurring kidney stone symptoms, of which only one was admitted. Thus, it can be inferred that most of the patients were stable and correctly diagnosed during their initial visits and that appropriate level of treatment was provided. Therefore, the recommendations to use CT scan WO contrast along with the options to order other tests (CT scan WWO and CT scan W contrast) based on clinical judgement may not only provide appropriate level of clinical effectiveness but also a faster and more efficient kidney stone diagnosing protocol.

With average $3 / 4$ to 1 hour saving in CT order to report times, about 42 minutes saving in room to discharge time, and an additional $37 \%$ of patients with suspicion of kidney stone shifting to CT scan WO contrast will lead to additional ED capacity to treat about 32 to 43 ED routine patients with average LOS of 250 minutes on annual basis for a 50,000 volume $\mathrm{ED}^{\mathrm{ii}}$. In addition, even

\footnotetext{
ii Note: Additional patients $=($ Annual suspicion of kidney stone volume*\% shift towards WO contrast*time saving)/expected LOS for routine patients $=(509 * 37 \% *(42$ or 57$)) / 250=32$ to 43 patients.
} 
though radiology services may experience reduced revenue due to of the ordering of fewer CT scans W and WWO contrast test, the additional capacity can be utilized for other revenue generating procedures. If the time-savings were translated to overall LOS and replicated to other common conditions (e.g., abdominal pain), management implications could include greater cost savings (i.e., lower overall costs, decreased charges, and better Value-Based Purchasing reimbursement capture), better quality (i.e., increased patient safety and satisfaction), and improved access (i.e., increased throughput, reduced overcrowding, and streamlining of backlogs).

Considering absence of standardization for diagnosing kidney stones, as well as distribution of utilization of CT scan modalities per hospital for suspicion of kidney stone, the results of the study can serve as a guideline for other EDs in both academic as well as community settings to streamline operations and reduce ED overcrowding. To further strengthen the kidney stone protocol, additional analysis is required that evaluates not only $\mathrm{CT}$ scan modalities, but also other diagnostic techniques such as ultrasound testing and Intravenous Urography.

\section{References}

Agency for Healthcare Research and Quality. (1995 (revised 2011)). ACR appropriateness criteria ${ }^{\circledR}$ acute onset flank pain - suspicion of stone disease. Retrieved from

http://www.guideline.gov/content.aspx $? \mathrm{id}=3$ 2639

Burt, C. W., \& McCaig, L. F. (2006). Staffing, capacity, and ambulance diversion in emergency departments: United states, 2003-04. Advance Data, (376)(376), 1-23.
Foster, G., Stocks, C. \& Borofsky, M. (2012). Emergency department visits and hospital admissions for kidney stone disease, 2009. HCUP statistical brief \#139. july 2012. agency for healthcare research and quality, rockville, MD.

Graham, A., Luber, S., \& Wolfson, A. B. (2011). Urolithiasis in the emergency department. Emergency Medicine Clinics of North America, 29(3), 519-538. doi:10.1016/j.emc.2011.04.007

King, D. L., Ben-Tovim, D. I., \& Bassham, J. (2006). Redesigning emergency department patient flows: Application of lean thinking to health care. Emergency Medicine Australasia, 18(4), 391-397.

Kinsman, L., Champion, R., Lee, G., Martin, M., Masman, K., May, E., . . . Zalstein, S. (2008). Assessing the impact of streaming in a regional emergency department. Emergency Medicine Australasia : EMA, 20(3), 221-227. doi:10.1111/j.17426723.2008.01077.x

Lotan, Y. (2009). Economics and cost of care of stone disease. Advances in Chronic Kidney Disease, 16(1), 5-10. doi:10.1053/j.ackd.2008.10.002

Marsh, A., Anderson, W., Bastani, A., \& Bastani, A. (2004/10). Decreasing emergency department wait times for available inpatient beds by removing artificial variation. Annals of Emergency Medicine, 44(Supplement 1), S28-S29.

MedlinePlus.Kidney stones.

Nash, K., Zachariah, B., Nitschmann, J., \& Psencik, B. (2007). Evaluation of the fast track unit of a university emergency department. Journal of Emergency Nursing, 33(1), 14-20. 
Oakley, E., \& Braitberg, G. (2005). Processes and impediments in moving patients from the emergency department: Pilot study. Emergency Medicine Australasia, 17(3), 266-273.

Pines, J. M. (2009). Trends in the rates of radiography use and important diagnoses in emergency department patients with abdominal pain. Medical Care, 47(7), 782786. doi:10.1097/MLR.0b013e31819748e9

Terris, J., Leman, P., O'Connor, N., \& Wood, R. (2004). Making an IMPACT on emergency department flow: Improving patient processing assisted by consultant at triage. Emergency Medicine Journal, 21(5), 537-541.

Westphalen, A. C., Hsia, R. Y., Maselli, J. H., Wang, R., \& Gonzales, R. (2011). Radiological imaging of patients with suspected urinary tract stones: National trends, diagnoses, and predictors. Academic Emergency Medicine : Official Journal of the Society for Academic Emergency Medicine, 18(7), 699-707. doi:10.1111/j.1553-2712.2011.01103.x; 10.1111/j.1553-2712.2011.01103.x

Wilson, M. J., \& Nguyen, K. (2004). Bursting at the seams: Improving patient flow to help America's emergency departments. Retrieved from http://urgentmatters.org/media/file/reports UM_WhitePaper_BurstingAtTheSeams.pdf 